\title{
A RICCATI SOLUTION FOR BURGERS' EQUATION*
}

BY ERVIN Y. RODIN (Washington University)

\begin{abstract}
In the last few years a one-dimensional, time-dependent and nonlinear approximation to the Navier-Stokes equations, (1) below, has found applications in fields as diverse as number theory, gas dynamics, heat conduction, elasticity, etc. Probably the most important reason for this is that the complete and explicit solution of this equation became known in 1950. That solution, however, applies only to the homogeneous part of Eq. (1). In an attempt to tackle the nonhomogeneous case, we relate Eq. (1) to a Riccati equation, through a similarity transformation. Via this route, it is shown that solutions to the nonhomogeneous equation can be obtained.
\end{abstract}

1. The homogeneous part of the nonlinear partial differential equation for $v=$ $v(x, t)$,

$$
v_{t}+v v_{x}=\delta v_{x x}+F(x, t), \quad \delta>0 \quad \text { (constant) }
$$

often called Burgers' equation [1], is solved [2], [3] by

$$
v=-2 \delta \frac{\partial}{\partial x}[\ln \theta]
$$

where $\theta$ itself is any positive solution of the linear heat equation,

$$
\theta_{t}=\delta \theta_{x x} \text {. }
$$

The nonlinear ordinary differential equation for $u=u(x)$,

$$
u^{\prime}=f(x)+g(x) u+h(x) u^{2},
$$

known as the generalized Riccati equation, can similarly be solved by an expression very much like (2):

$$
u=-\frac{1}{h(x)} \frac{d}{d x}[\ln \phi]
$$

Here $\phi$ is any solution of a linear equation, which with a further transformation can be brought into the form (compare Eq. (3))

$$
\Phi^{\prime \prime}+P(x) \Phi=0,
$$

with $P$ given as a specified combination of the functions $f, g$ and $h$.

It may be of some interest therefore to see how (1)-(3) can be reduced to (4)-(6); particularly since the method will allow us to obtain simple solutions for (1), when the forcing function $F \neq 0$ is of a special form.

2. We shall achieve the reduction of the partial differential equation (1) to an ordinary differential equation by means of a similarity transformation. Specifically, we begin by introducing a function $\psi$, defined by

$$
v=\psi_{x} .
$$

\footnotetext{
* Received December 15, 1968. This work was supported by NASA Grant NLG 26-008-006.
} 
Then (1), with $F \equiv 0$, can be written in terms of $\psi$ as

$$
\psi_{x t}+\psi_{x} \psi_{x x}=\delta \psi_{x x x} .
$$

We are assuming here all the necessary continuity properties for the functions used: in particular, $\psi_{x t}=\psi_{t x}$. Thus, (8) can be integrated to yield

$$
\psi_{t}+\frac{1}{2} \psi_{x}^{2}=\delta \psi_{x x} .
$$

We now take the form

$$
z=x^{2} / t
$$

for our similarity variable $z$, and assume that

$$
\psi(x, t)=\psi=f(z)=f\left(x^{2} / t\right) .
$$

Then (9) becomes

$$
-\frac{x^{2}}{t^{2}} f^{\prime}+\frac{1}{2}\left(\frac{2 x}{t}\right)^{2}\left(f^{\prime}\right)^{2}=\delta\left[\frac{4 x^{2}}{t^{2}} f^{\prime \prime}+\frac{2}{t} f^{\prime}\right],
$$

an expression which, after a slight rearrangement and the use of (10), gives our Riccati equation in $f^{\prime}$ :

$$
f^{\prime \prime}=\frac{1}{2 \delta}\left(f^{\prime}\right)^{2}-\left(\frac{z+2 \delta}{4 \delta z}\right) f^{\prime} .
$$

Note that the $h(x)$ of (4) is $1 / 2 \delta$ here; so that the solution (5) will have, as its leading factor, $(-2 \delta)$. This is of course in conformity with the form (2).

One solution of (12) is an identically constant one. We shall find the other directly. Letting

$$
f^{\prime}(z)=-2 \delta / y(z)
$$

Eq. (12) becomes

$$
y^{\prime}-\frac{z+2 \delta}{4 \delta z} y=1,
$$

which has a solution containing an arbitrary constant $A$ :

$$
y(z)=A \sqrt{ } z e^{z / 4 \delta}+\int_{1}^{z} \sqrt{\frac{z}{z_{1}}} e^{\left(z-z_{1}\right) / 4 \delta} d z_{1} .
$$

From (15) and (13) we get

$$
f^{\prime}(z)=-2 \delta \frac{\frac{1}{\sqrt{ } z} e^{-z / 4 \delta}}{A+\int_{1}^{z} e^{-z_{1} / 4 \delta} \frac{d z_{1}}{\sqrt{ } z_{1}}} .
$$

On the other hand, since

$$
\frac{\partial f}{\partial x}=\frac{d f}{\partial z} \frac{\partial z}{\partial x}=f^{\prime}\left(\frac{2 x}{t}\right),
$$


relations (7) and (10) yield

$$
v(x, t)=\frac{-4 \delta}{\sqrt{ } t} \frac{\exp \left[-x^{2} /(4 \delta t)\right]}{A+\int_{1}^{x^{2} / t} \exp \left[-z_{1} /(4 \delta)\right] \frac{d z_{1}}{\sqrt{ } z_{1}}} .
$$

3. Up to this point we had no occasion to use our knowledge concerning Riccati equations. Let us consider now, however, the nonhomogeneous equation (1):

$$
v_{t}+v v_{x}=\delta v_{x x}+F(x, t) .
$$

Its solution, as can be shown quite easily, is still given by (2); now, however, $\theta$ has to satisfy

$$
\theta_{\imath}=\delta \theta_{x x}+\left(\int^{x} F(\xi, t) d \xi\right) \theta .
$$

The heat equation $\left(3^{\prime}\right)$ is still linear, but, as a practical matter, it is rather difficult to obtain solutions for it in a relatively easily analyzable form. We turn therefore to the Riccati route.

Extending the similarity assumption to the function $F$ in the form

$$
t \int^{x} F(\xi, t) d \xi=G_{1}\left(\frac{x^{2}}{t}\right),
$$

introducing $\psi$ as in (7), as well as the assumptions (10), we can put Eq. (9) into the form

$$
\psi_{t}+\frac{1}{2} \psi_{x}^{2}=\delta \psi_{x x}+G_{1}(z) / t
$$

and the analogue of Eq. (12) becomes

$$
f^{\prime \prime}=\frac{1}{2 \delta}\left(f^{\prime}\right)^{2}-\left(\frac{z+2 \delta}{4 \delta z}\right) f^{\prime}-\frac{1}{4 \delta z} G_{1}(z) .
$$

The next step is the transformation of (19) to the form (8). Let us therefore introduce a function $E=E(z)$, such that

$$
f^{\prime}(z)=-2 \delta \frac{E^{\prime}(z)}{E^{\prime}(z)} .
$$

Eq. (19) becomesi

$$
E^{\prime \prime}+\frac{z+2 \delta}{4 \delta z} E^{\prime}=\frac{1}{8 \delta^{2} z} G_{1}(z) E .
$$

This is the crucial equation to be solved; it is the analogue of $\left(3^{\prime}\right)$. Clearly, for large classes of functions $F$ (thus, $G_{1}$ ), it is possible to obtain series solutions for (20). For a considerably more limited class one has closed form solutions; indeed, it is possible to use here lists of solutions of differential equations such as Murphy's [4].

4. Let us consider now one special case: when the foreing function $F$ is linear in $x$. We take, specifically

$$
F=\frac{1}{t}\left[\frac{a_{1}}{\sqrt{ } t}+\frac{a_{2} x}{t}\right] .
$$


Then from

$$
\begin{aligned}
G_{1}\left(\frac{x^{2}}{t}\right) & =t \int^{x} \frac{1}{t}\left[\frac{a_{1}}{\sqrt{ } t}+\frac{a_{2} \xi}{t}\right] d \xi \\
& =a_{1} \frac{x}{\sqrt{ } t}+\frac{a_{2}}{2} \frac{x^{2}}{t}=a_{1} \sqrt{ } z+\frac{a_{2}}{2} z
\end{aligned}
$$

Thus (20) becomes

$$
E^{\prime \prime}+\left(\frac{1}{4 \delta}+\frac{1}{2 z}\right) E^{\prime}=\left[\frac{a_{1}}{8 \delta^{2} \sqrt{ } z}+\frac{a_{2}}{16 \delta^{2}}\right] E .
$$

In terms of the variable

$$
u=\sqrt{ } z
$$

Eq. (23) is

$$
\frac{d^{2} E}{d u^{2}}+\left(\frac{u}{2 \delta}\right) \frac{d E}{d u}=\left(\frac{a_{1} u}{2 \delta^{2}}+\frac{a_{2} u^{2}}{4 \delta^{2}}\right) E,
$$

whose solution, in terms of the arbitrary constants $A$ and $B$, is

$$
E(u)=e^{\alpha u^{2}+\beta u}\left\{A+B \operatorname{erf}\left[\left(2 \alpha+\frac{1}{4 \delta}\right) u\right]\right\} .
$$

The constants $\alpha$ and $\beta$ are given in terms of $a_{1}$ and $a_{2}$ of (21), as well as $\delta$ :

$$
\begin{aligned}
& \alpha=\frac{-1 \pm \sqrt{1+4 a_{2}}}{8 \delta} \\
& \beta=\frac{ \pm a_{1}}{\delta \sqrt{1+4 a_{2}}} .
\end{aligned}
$$

Notice here the definite limitations on the magnitude of $a_{2}$, which is the coefficient of the only term containing $x$ in (21). Perhaps it also ought to be mentioned that the relevant magnitude of $\delta$, for gas dynamics, is around $10^{-4}$; so that $|\alpha|$ and $|\beta|$ can be quite large. As far as the \pm ambiguity is concerned: it will be seen to disappear. Restoring now $\sqrt{ } z=u$ in (26), we have

$$
L(z)=e^{\alpha_{z+\beta} \sqrt{ }_{z}}\left\{A+B \operatorname{erf}\left[\left(2 \alpha+\frac{1}{4 \delta}\right) \sqrt{ } z\right]\right\} .
$$

Thus the solution $v$ is obtained from $\left(26^{\prime}\right)$ as in (17); it is

$$
\begin{aligned}
v(x, t) & =f^{\prime}(z) \frac{\partial z}{\partial x}=-2 \delta \frac{d}{d z}[\ln E(z)] \frac{\partial z}{\partial x} \\
& =-4 \delta \frac{x}{t} \frac{\frac{2}{\sqrt{ } \pi} e^{-(2 \alpha+1 / 4 \delta) x^{2} / t}+\left(\alpha+\frac{\beta}{2 x} t\right)\left\{\bar{A}+\operatorname{erf}\left[\left(2 \alpha+\frac{1}{4 \delta}\right) \frac{x}{\sqrt{ } t}\right]\right\}}{\bar{A}+\operatorname{erf}\left[\left(2 \alpha+\frac{1}{4 \delta}\right) \frac{x}{\sqrt{ } t}\right]}
\end{aligned}
$$

In (28), the two constants coalesce into one; we denote it by $\bar{A}$.

We can now settle the \pm ambiguity which arose in (27). Observing that if $\alpha=\beta=0$, 
then (28) reduces to the solution of the homogeneous form of (1), we choose the + signs in (27). This will make $\alpha=\beta=0$ when $a_{1}=a_{2}=0=F$.

5. In conclusion, we observe that we obtained our solutions without recourse to the general formula (1-2). In doing so, we lost a great deal in the possibility of satisfying some very natural boundary and/or initial conditions for Eq. (1). On the other hand, we gained (a) an ability to solve, in a somewhat simpler fashion, the nonhomogeneous equation, and (b) an insight into the nature of the general solution of (1), which, if it were not available, could possibly be guessed at from an expression such as (17). Thus, if this method be found applicable to other equations for which the general solutions are not yet known, then the similarity solutions might point a way towards the general ones. Finally, we note that (28) itself is believed to be a new result.

\section{REFERENCES}

[1] J. M. Burgers, Advances in applied mechanics, Academic Press, New York, 1948

[2] E. Hopf, Commun. Pure Appl. Math. 3, 201 (1950)

[3] J. D. Cole, Quart. Appl. Math. 9, 225 (1951)

[4] G. M. Murphy, Ordinary differential equations and their solutions, D. Van Nostrand, Princeton, 1960 